\title{
Design and Prototype of the high voltage In-Service- Tests on the superconducting magnet system of Wendelstein 7-X
}

\author{
F. Füllenbach, Th. Rummel, Th. Mönnich, E. Köster
}

\begin{abstract}
The stresses induced during the energizing and deenergizing process of the Wendelstein $7-X$ super conducting magnet system could cause cracks in the embedding of the winding packages and consequently a ground fault that would not be detected during normal operation as the operating voltage of the magnet is system is too low. With a ground fault present during a fast discharge, the middle point grounding needed to half the voltage could be rendered useless and one coil group could be stressed with the full voltage of up to $4 \mathrm{kV}$ which would endanger the high voltage integrity of the whole superconducting magnet system. To prevent such undesirable occasions, the insulation of the superconducting magnet system shall be monitored by a high voltage In-Service-Test. With the In-ServiceTest a voltage of $2.5 \mathrm{kV}$ can be applied during the operation of the magnets especially after significant changes in the coil current. The measured leak current can then be used to determine any changes in the quality of the insulation. In a first step the feasibility of such a system has been evaluated with a simulation model of one coil group with Simplorer $^{\odot}$. The next step was to find a design which can be implemented in the given magnet power supply system and to build and test a prototype for final confirmation of the concept.

The paper describes the concept design of the high voltage InService test together with the design, manufacturing and test of a first prototype.
\end{abstract}

Index Terms-Wendelstein 7-X, superconducting coils, power supplies, high voltage test

\section{INTRODUCTION}

$\mathrm{T}$ HE SUPERCONDUCTING MAGNET SYSTEM OF W7-X consists of 50 nonplanar and 20 planar coils in stainless steel housing. During energizing and de-energizing operations forces of up to $1,5 \mathrm{MN}$ will lead to deformations in the range of $35 \mathrm{~mm}$ of the coil housing thereby stressing the glass fiber reinforced epoxy insulation [1]. The 70 superconducting magnets are divided into 7 coil groups of ten equal coils in series in each group. Every group is powered by an individual power supply. The seven power supplies are twelve pulsing rectifiers built upon M3 thyristor groups in parallel with interconnecting interphase transformers [2]. The five power

Manuscript received August 1, 2013.

F. Füllenbach, Th. Rummel, Th. Mönnich, and E. Köster are with the MaxPlanck-Institute for Plasma Physics, 17491 Greifswald, Germany, (corresponding author phone: +49 383488 2776; fax: +49 383488 2709; email: frank.fuellenbach@ipp.mpg.de). supplies feeding the non planar coil groups shall be equipped with the system described in the following paper. The planar coil groups will suffer less mechanical stress during operation. Also the high voltage created in these coil groups during a fast discharge is significantly smaller than in the non planar coil groups. Therefore the power supplies for these two coils groups are not to be considered for the described application.

For monitoring the insulation of the superconducting magnets a high voltage In-Service-(IS) test system will be integrated in the power supply system of the W7-X. This IStest system shall measure the leak currents generated by high voltage during operation every time after the coils are stressed due to a significant change in the current they have to carry. The changes in the leak currents shall give an insight in the probably changing quality or even serious damage of the insulation between coil winding and coil housing to ground.

In preliminary studies including a simulation of the principle function of the IS test system during normal operation as well as during fast discharges in the magnet system, the feasibility of the IS-test system has been investigated and the main parameters have been fixed [3]. For further proof of principle a series of tests has been performed including one with the power supply for the superconducting magnets before fixing a first conceptual design for a prototype. Figure 1 shows the principle circuit for the coil power supply system and the IStest system included in the ground path and connected to the coil housing over the magnet system's support structure. Also the main characteristic parameters of nominal voltage and current, grounding resistors and the IS-test voltage are displayed.

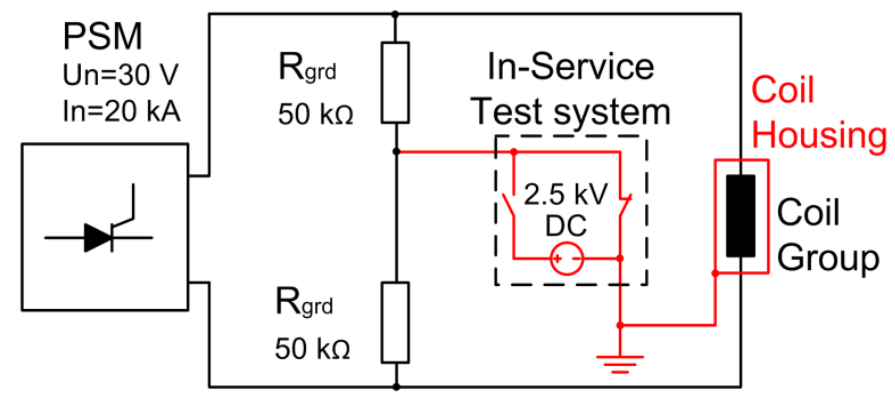

Fig. 1. Principle sketch of the IS-test system integrated into the power supply of the superconducting magnets consisting of the power supply module (PSM) and the middle point grounding resistors $\left(\mathrm{R}_{\mathrm{grd}}\right)$ 


\section{PRELIMINARY TESTS}

Three different tests were performed to demonstrate that a very small leak current can be detected while high currents are applied to the coils. Similarly it had to be proven that the high voltage would not have a disturbing feedback on the power supply, especially on the rectifier and the fast discharge system required for coil protection. The first step was a very small laboratory set-up followed by a small scale test with a high current power supply leading to a full scale test with the dedicated power supplies for the superconducting magnet system. For all three tests accompanying simulations in Simplorer $^{\odot}$ have been carried out to verify the test results.

\section{A. Laboratory Test}

A set of six coils with an inductance of $\mathrm{L}=600 \mu \mathrm{H}$ and resistance at room temperature of $\mathrm{R}_{20^{\circ}}=19,3 \mathrm{~m} \Omega$ acting as the magnet system was operated at $2,7 \mathrm{~V}$ DC and $10 \mathrm{~A}$ with a laboratory power supply while the small scale IS-test voltage is set to $15 \mathrm{~V} \mathrm{DC}$ with another laboratory power supply. Grounding resistors as shown in fig. 1 were set to $270 \Omega$ each limiting the current to ground to about $5 \mathrm{~mA}$. Resistors in range of $12 \Omega$ to $100 \mathrm{k} \Omega$ were added between the coil terminals to ground simulating degradation of insulation. The leak currents were measured in a range of $100 \mathrm{~mA} @ 12 \Omega$ to about $10 \mathrm{~mA} @ 1 \mathrm{k} \Omega$. With higher resistances the additional leak current became negligible small as the insulation resistance of the coils was not much higher. Also the accompanying simulations verified these values.

\section{B. Small scale test}

The next step was to operate an IS-test system mock up with one of the W7-X control coils power supplies [2]. The control coil power supply is a four quadrant converter with a line commutated DC link rectifier and operates the control coil with about $12 \mathrm{~V} \mathrm{DC}$ at $2500 \mathrm{~A}$. The IS-test voltage was set to $100 \mathrm{~V}$ in order to keep the test set up as simple as possible with regards to safety. Leakage resistors were dimensioned from $47 \Omega$ to $10 \mathrm{k} \Omega$ allowing leaking currents to ground of up to 2 A. Three dummy loads with an inductance of $\mathrm{L}=180 \mu \mathrm{H}$ and a resistance at room temperature of $\mathrm{R}_{20^{\circ}}=3,9 \mathrm{~m} \Omega$ coils representing a control coil each were connected in series in order to simulate ground faults at different stages of the test set up. For the small scale test these coils were operated at 500 A. Again the test results were similar to the simulations as expected. The power supply including the 4 quadrant converter as well as the DC link rectifier did not show any influence by the IS-test voltage applied to it.

\section{Full scale test}

Before using high voltage on the power supply for the superconducting magnets it was carefully checked that all components of the power supply that will be raised to high voltage against ground are designed for it. Not all the parts of the power supply will be included in the fast discharge circuit during a fast discharge which is high voltage proven and therefore are not necessarily adequate for the IS-test voltage. In a next step an existing high voltage source was operated inside the grounding path to verify that no component has been overseen in this evaluation process. No current operations were done during this test.

After that the dedicated high voltage source that has been already been chosen for the IS-test system was operated at the power supply of the superconducting coils. As the magnet system is not already connected to the power supply it was operated in short circuit because the main goal of this test was to verify that there are no undesired feedbacks of the InService test into the power supply. One high voltage resistor with $63 \mathrm{k} \Omega$ was connected between one of the high current bus bars and ground. With this simulated ground fault a leakage current to ground of about $30 \mathrm{~mA}$ was expected. Again the test results were in compliance with the simulated values and the power supply did not show any undesired behavior during the IS-test. Following this test the decision was made to design a first prototype.

\section{CONCEPTUAL DESIGN}

The main focus in the concept design was dedicated to the switching technology for commutating the high voltage source into the ground path. The basic parameters for choosing the technology were high reliability as the IS test system has to be operated for a whole week without having access to the hardware, easy maintainability and their operability under the influence of magnetic fields in the range of up to $10 \mathrm{mT}$ as the power supplies are located quit close to the Stellerator. Also cost factors had to be considered. Therefore it was decided to use ordinary high voltage switches from SPS that are already in use for the high voltage power supply system of the glow discharge units of the W7-X [4]. These switches work reliable at DC voltage of up to $9 \mathrm{kV}$ and can carry currents of $30 \mathrm{~A}$. For the present application they have been tested in magnetic fields. The switches were operated with no measureable influence in their switching behavior at up to $15 \mathrm{mT}$. At higher magnetic fields the switching time started to increase from about $28 \mathrm{~ms}$ to about $94 \mathrm{~ms}$ at $36 \mathrm{mT}$. Above $36 \mathrm{mT}$ the switches started failing sporadically. The results are considered to include enough margins to operate the switches reliable at $10 \mathrm{mT}$. Also the switching times have been considered to assure that in case of a detected quench in the magnet system the IS-test is immediately shut off before the fast discharge of the magnet system starts.

While the IS-test voltage source is included in the ground path it is inevitable to have the high resistance of the IS-test voltage power supply in the ground path. Nevertheless the complete disruption of the ground during switching operation even for few milliseconds should be avoided. Therefore a special switching topology was designed to never completely interrupt the ground path. In this topology the IS-test power supply is connected in parallel to the closed grounding path and therefore short circuited while having a small voltage set point at the output. This is no problem as modern switching power supplies can easily be operated in short circuits with nominal currents even in steady state.

The second important component to be chosen is the IS-test high voltage power supply. It has to deliver $2.5 \mathrm{kV}$ DC while 
the expected leakage currents at normal operation will be in the range of several $\mathrm{mA}$ mostly driven by the conductivity of the cooling water of the power supply that will range from 1 to $10 \mu \mathrm{S} / \mathrm{cm}$. Should a complete breakdown of the insulation of the coils happen and a low resistive contact to the ground system appears, the leakage current will be limited by the grounding resistors to a maximum of less than $100 \mathrm{~mA}$. Table 1 gives an overview of the design parameters for the power supply.

TABLE I

DESIGN PARAMETERS FOR THE HIGH VOLTAGE SUPPLY

\begin{tabular}{ll}
\hline \multicolumn{1}{c}{ Parameter } & \multicolumn{1}{c}{ Value } \\
\hline output voltage & $\geq 2.5 \mathrm{kV}$ \\
output current & $20 \mathrm{~mA} \ldots 100 \mathrm{~mA}$ \\
integrated current feedback & $\pm 0.1 \mathrm{~mA}$ \\
measurement precision & \\
short circuit behavior & short circuit proof, operable at \\
& nominal current in short circuit \\
communication interface & Profibus DP \\
grounding & 0 V potential floating to ground
\end{tabular}

The chosen high voltage source is a power supply from $F U G$ type HCP140-3500 with an output voltage of $3,5 \mathrm{kV}$ and a maximum output current of $40 \mathrm{~mA}$. The integrated current feedback measurement precision is $10^{-4}$ therefore $4 \mu \mathrm{A}$. A Profibus DP interface is one of the several standardized options that can be included off the shelf.

Another important design criterion is the personnel and machine safety. Even if the high voltage will mainly be induced during operation of the machine while no personnel is in the machine hall, for test and maintenance purposes the IS test system has to be operated together with the coils also in shut down times of the machine. In case of an insulation failure in the magnet system, the high voltage could reach all parts of the machine and endanger personnel in the machine hall. Therefore it has to be assured that while the IS test system is not needed an accidental activation must not happen. To do so, two hardwired interlocks are established into the main circuit. One to bypass the switch gear in the grounding path and one to connect the HV output of the IS test voltage power supply to ground.

Fig 2 shows the main circuit diagram of the final design for one In-Service Test system with the high voltage supply (HV supply) with the integrated current feedback measurement, the switch gear (K1...K5), self test resistor (R1), the hardware interlocks (X1.1..X2.2), $230 \mathrm{~V}$ grid supply and the PLC inside a cabinet.

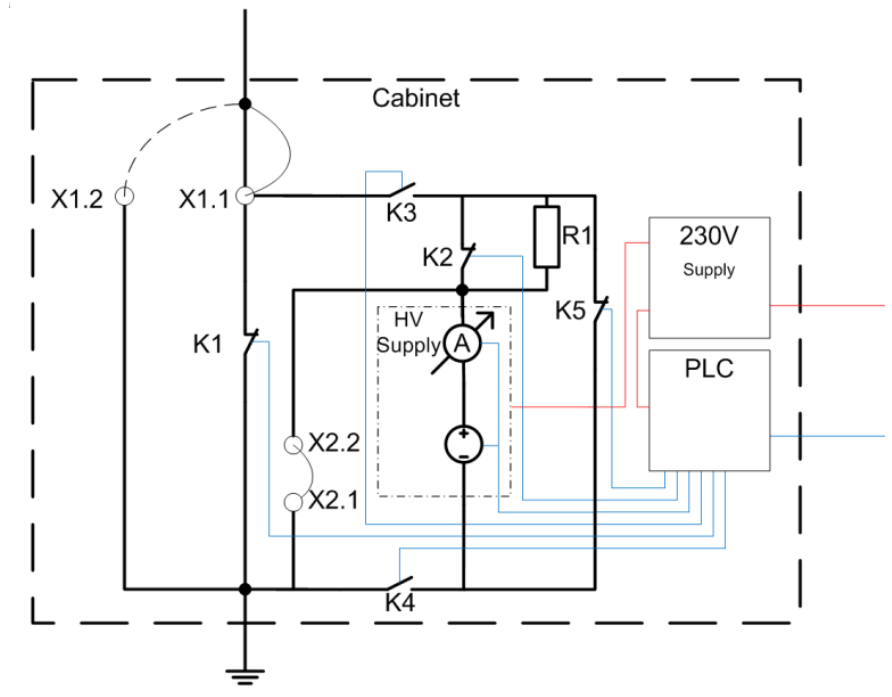

Fig. 2. main circuit of the IS-test system with HV source, switch gear (K1 ...5), hard wired safety interlocks (X1.1...X2.2), PLC and mains supply

To verify the proper functionality of the IS-test power supply a test circuit shall be integrated to assure that the communication, the set point processing and the current feedback signal of the HV power supply are reasonable. This test circuit is a simple resistor that can be connected to the output of the IS test power supply to operate at $2.5 \mathrm{kV}$ and creates a full current feedback of $40 \mathrm{~mA}$. Figure 3 shows the full switching sequence together with the IS-test voltage output set point starting with the self test (1.) over to the preparation of the commutation (2.) before finally switching the HV power supply into the ground path and starting the IS test (3.).

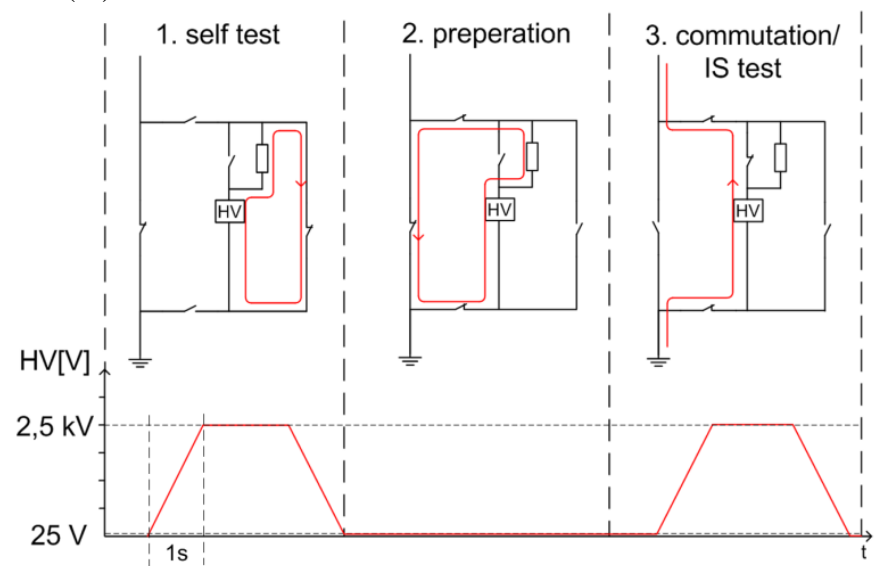

Fig. 3. switching sequence (top) with current path (red line) together with the HV voltage output over time (bottom)

\section{THE PROTOTYPE}

The development of the prototype was done in two separated steps:

- Designing the switch gear for commutation with a hardwired control by link relays and integration of the high voltage power supply together with a manually operated user interface and establishing the hardware for the interlock plugs and sockets.

- Integrating the remote operation via PLC into the given 
design together with interlock and safety signals.

\section{A. First Step Prototype}

During the design of the first step prototype features like placement of the IS Test in the power supply area, cabinet size, orientation of cable feed troughs and the Human Machine Interface (HMI) for manual operation have been developed. Special care has been taken for the interlock signals that need to be created for the two different grounding states of the IS test system. This can be a "safe" status were the output of the $\mathrm{HV}$ power supply is grounded, the ground path is fixed to ground and the switchgear of the IS test system is bypassed or the "operation" status in which the switchgear can be operated. For doing so a high voltage plug with auxiliary read out contacts has been developed with the company LEMO. Two of these plugs are used, one to bypass the switch gear (refer to X1.1 and 1.2 in Fig. 2) and one to ground the output of the high voltage source (refer to X2.1 and 2.2 in Fig. 2.). For each plug there is a safety and a operation socket which is associated with the auxiliary read out contacts. Only if both plugs are inserted in the safety socket the IS-test system is considered save and clearance can be given by the overall safety control for access to the experiment building. Only if both plugs are inserted in the operation socket supply voltage can be given to the IS-test system.

After completion the prototype was successfully tested together with one power supply module for the superconducting magnets with regards to switching technology, safety interlocks and current measurement feedback.

\section{B. Second Step Prototype}

For the second step prototype the manual switchgear control has been replaced by a local PLC interface module to be connected to one dedicated PLC CPU which will be programmed to control all the five local PLC interface modules in the IS-test systems. The PLC hardware is completely based upon Siemens $S 7$ technology. The control software has been developed with respect to the two different control modes:

- autonomous control

- superordinated control

While under autonomous control the two different access modes have to be established as well:

- local access

- remote access

Furthermore the cabinet design was finalized with respect to all interfaces giving the basis for the series production. Figure 4 shows the second step prototype with its manual HMI. This prototype has been thoroughly tested with respect to the control functionality in conjunction with the dedicated PLC for the IS test system.

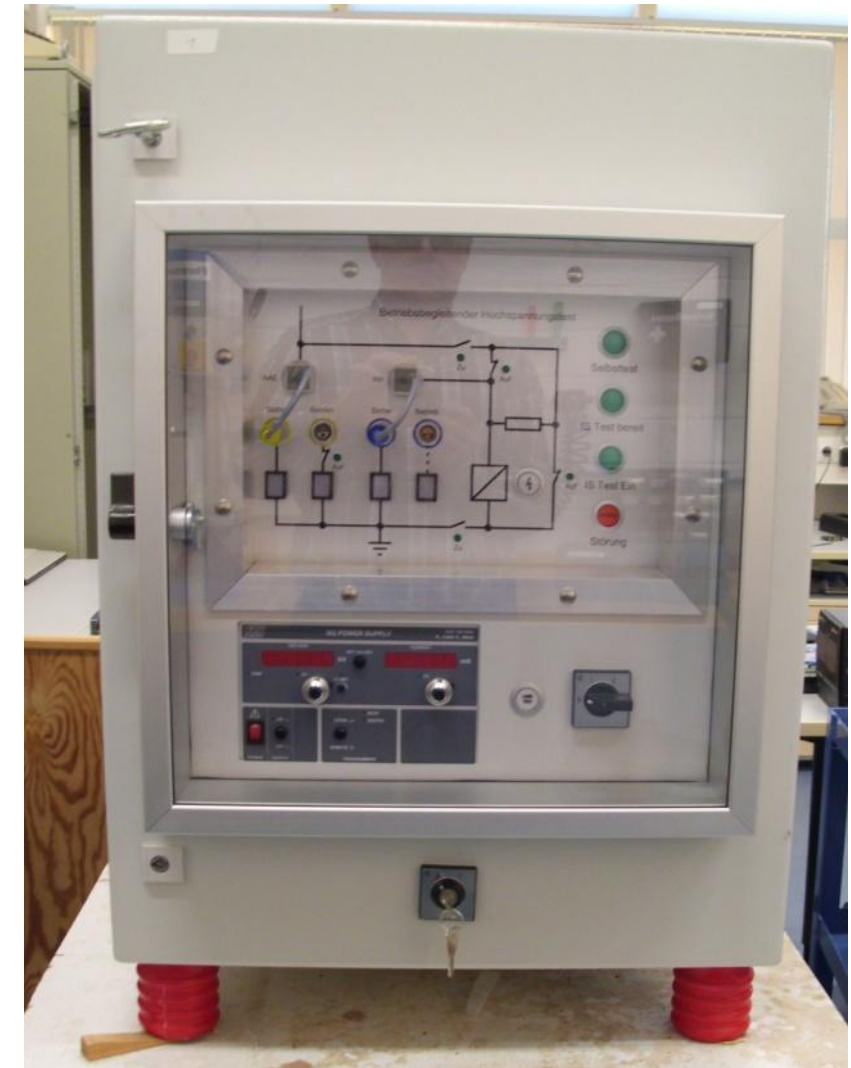

Fig. 4. Second step prototype with manual operation HMI

\section{TEST RESUlts ON PROTOTYPE}

The first step prototype has been tested according to the standard rules for electrical cabinets DIN VDE 0100 part 600 and with respect to high voltage immunity. Following a heat run, measuring the heat dissipation caused by the self test resistor has been performed. Finally the first step prototype was used for a repeated test described in section $1 \mathrm{~A}$ where the full functionality was proven.

With the second step prototype all controls, maneuvers and feedbacks in the different access- and control modes have been tested as well as the interlocks between them. After evaluating the test results decision was made that the given design of hard- and software can be manufactured in series.

\section{SUMMARY}

After proving the feasibility of the IS-test concept with three different test arrangements, two prototypes of the IS-test system, one for proofing the technology of the switch gear and one with the final control topology already included have been developed, manufactured and tested successfully. An appropriate design for commutating technology, safety measure and integration has been developed as well as the identification of suitable components for the different stages of the system.

The IS-test system is right now under construction at IPP and after installation and integration a test campaign in conjunction with the control system of the power supply will take place. 


\section{ACKNOWLEDGMENT}

The authors would like to thank Michel Huguet for inspiration of the principle technology and also all the involved colleagues in IPP for their work and co-operation.

\section{REFERENCES}

[1] V. Bykov, et al., Structural analysis of W7-X: Overview, Fusion Eng. Des. 84, 215-219 (2009)

[2] Th. Rummel, et al, "Power supplies for the Wendelstein 7-X stellarator", Fusion Engineering and Design, 66-68 (2003), pp. 11151118

[3] Füllenbach, F.; Rummel, T.; Mönnich, T.; Feasibility Study of a High Voltage In-Service-Tests on the Superconducting Magnet System of Wendelstein 7-X. IEEE Transactions on Applied Superconductivity 22 (3), (2012)

[4] Th. Rummel, et al, "The power supplies for the glow discharge electrodes in Wendelstein 7-X", Fusion Engineering and Design, 86 (2011) 1562-1565 